\title{
Overexpression of GNAO1 correlates with poor prognosis in patients with gastric cancer and plays a role in gastric cancer cell proliferation and apoptosis
}

\author{
ZHEN LIU $^{1 *}$, JUN ZHANG $^{2 *}$, LIJUN WU $^{2}$, JIE LIU $^{2}$ and MINGQING ZHANG ${ }^{1}$ \\ ${ }^{1}$ Department of Digestive Diseases, Southeast Hospital, Xiamen University, Zhangzhou, Fujian; \\ ${ }^{2}$ Department of Digestive Diseases, Huashan Hospital, Fudan University, Shanghai, P.R. China
}

Received September 4, 2013; Accepted December 11, 2013

DOI: $10.3892 /$ ijmm.2013.1598

\begin{abstract}
Guanine nucleotide-binding protein (G protein), alpha activating activity polypeptide $\mathrm{O}$ (GNAO1) encodes an alpha subunit of the heterotrimeric guanine nucleotide-binding proteins $(\mathrm{G} \alpha \mathrm{o})$, which plays a significant role in the development of various types of cancer, including breast cancer and hepatocellular carcinoma. However, its role in gastric cancer (GC) has not yet been elucidated. In the current study, the expression of Gao was analyzed by immunohistochemistry in paraffin-embedded tissue sections from 70 patients with GC and its cellular role was examined by small interfering RNA (siRNA)-mediated downregulation. The overexpression of GNAO1 (scores of 2 or 4) was observed in 44 of the 70 patients with GC (62.9\%) and the expression of Gao significantly correlated with the overall survival of the patients with GC after surgery. The median survival rate of patients with a negative or positive expression of Gao was 61 or 27 months, respectively ( $\mathrm{P}=0.033)$. The silencing of GNAO1 in GC cells by RNA interference markedly inhibited the proliferation of GC cells and promoted apoptosis by increasing the accumulation of the pro-apoptotic proteins, Puma and Bim, possibly mediated by extracellular signal-regulated kinase 1 and 2 (ERK1/2). This further confirmed the clinical significance of GNAO1, which was overexpressed in GC tissues. To the best of our knowledge, the present study is the first to demonstrate that GNAO1 is overexpressed in GC and that its overexpression correlates with poor prognosis, as it promotes GC cell viability.
\end{abstract}

Correspondence to: Professor Mingqing Zhang, Department of Digestive Diseases, Southeast Hospital, Xiamen University, 269 Middle Zhanghua Road, Zhangzhou, Fujian 363000, P.R. China

E-mail: zhangmq8084@hotmail.com

Professor Jie Liu, Department of Digestive Diseases, Huashan Hospital, Fudan University, 12 Middle Wulumuqi Road, Shanghai 200040, P.R. China

E-mail: jieliu@fudan.edu.cn

*Contributed equally

Key words: guanine nucleotide-binding protein (G protein), alpha activating activity polypeptide $\mathrm{O}$, gastric cancer, proliferation, apoptosis

\section{Introduction}

Gastric cancer (GC) is the fourth most commonly diagnosed cancer with the second highest mortality rate worldwide (1). Its incidence rate is the highest in Eastern Asia (2). Although substantial advances have been made in surgical techniques and chemotherapy, which have improved the effectiveness of treatment strategies for GC, the overall outcome for patients with GC has not changed significantly over the past few decades (3). Biotherapy, the fourth treatment option after surgery, chemotherapy and radiotherapy, includes gene therapy, which is a prospective new method that has developed in recent years for malignant cancers. Some biological prognostic markers which may aid in the understanding of disease pathogenesis have been identified, such as Krüppel-like factor 8 (4), excision repair cross-complementing gene 1 (ERCC1) (5) and Forkhead box protein M1 (FOXM1) (6). However, compared to other more extensively investigated cancers, such as breast, prostate and colon carcinomas, the molecular mechanisms involved in the pathologenesis of GC are poorly characterized, and the cure rate for patients with GC remains low, while the morbidity rate remains high (7). Hence, additional studies are required in order to enhance our understanding of the pathogenesis of GC and its biological features, along with newer clinically relevant molecular markers that can be used in GC.

Heterotrimeric guanine nucleotide-binding proteins (G proteins) are molecular switches that control signal transduction, and their dysregulation can promote oncogenesis. Gao, encoded by guanine nucleotide-binding protein (G protein), alpha activating activity polypeptide $\mathrm{O}$ (GNAO1), belongs to the family of G protein alpha subunits (8) and is abundantly expressed in neuronal tissue (9). Apart from the activation of second messenger molecules, G $\alpha$ o subunits can modulate the activity of transcription factors and thereby regulate gene expression (10-12). Moreover, recent studies have found that $\mathrm{G} \alpha$ o subunit alterations play a potentially more significant role in different types of cancer, such as breast cancer (13) and hepatocellular carcinoma (HCC) (14). With the use of mismatch repair detection (MRD) technology, a germline mutation at residue R243 was found to be associated with breast cancer (13). The first somatic mutation of GNAO1 (Gao) was described in breast cancer, and was proven to promote 
oncogenic transformation possibly through signal transducer and activator of transcription 3 (STAT3) signaling in vitro (15).

However, to date, the expression and possible roles of GNAO1 in GC have not been established. The aim of the present study was to analyzed the expression of GNAO1 protein in $\mathrm{GC}$ tissues and to determine the correlation between $\mathrm{G \alpha o}$ expression and the clinicopathological parameters of patients with $\mathrm{GC}$, as well as to investigate the effects of GNAO1 protein expression on the biological behavior of GC cells.

\section{Materials and methods}

Recruitment of patients with GC and tissue specimens. Tissue samples from 70 unrelated patients with primary GC, who were treated at Shanghai Huashan Hospital, Shanghai, China were obtained at the time of surgical resection between January and December 2005. The tumors and corresponding adjacent tissues were obtained after first having received patient informed consent under a protocol reviewed and approved by the Human Research Review Committee of Huashan Hospital, Fudan University, Shanghai, China. The tissues were fixed in paraffin for pathological diagnosis and immunohistochemical staining. Histopathological diagnosis of each neoplastic tissue was performed according to the World Health Organization criteria by the Department of Pathology, Huashan Hospital. Clinicopathological staging was determined based on the TNM classification. All patients with GC were confirmed by histological analysis, and tumor samples were examined to ensure that tumor tissue was present in $>80 \%$ of the specimens. Follow-up data were collected until December 2011 or until the patient became deceased, and the occurrence of metastasis and/ or local recurrence was recorded.

Immunohistochemistry. Immunohistochemical staining with rabbit anti-human GNAO1 polyclonal antibody (Proteintech Group, Chicago, IL, USA) was performed on 4-mm-thick sections of paraffin-embedded specimens. Briefly, following deparaffination and hydration, the slides were incubated in $0.3 \% \mathrm{H}_{2} \mathrm{O}_{2}$ for $10 \mathrm{~min}$ in order to block endogenous peroxidase before being boiled in citrate buffer $\mathrm{pH} 6.0$ for $15 \mathrm{~min}$. The slides were then incubated with rabbit anti-human GNAO1 polyclonal antibody (Proteintech Group) overnight at $4^{\circ} \mathrm{C}$ in a moist chamber. Subsequently, the specimens were washed 3 times in PBS and treated with a biotinylated goat antirabbit antibody at $37^{\circ} \mathrm{C}$ for $2 \mathrm{~h}$. The specimens were washed another 3 times in PBS and then treated with $\mathrm{ABC}$ reagent, followed by color development in 3,3'-diaminobenzidine tetrahydrochloride (both from Dako, Carpinteria, CA, USA). Finally, the slides were lightly counterstained with haematoxylin (Baso Diagnostics Inc., Zhuhai, China). All specimens were observed and photographed under a microscope. Duplicate sections were immunostained without exposure to primary antibodies and used as negative controls. The immunostaining pattern of GNAO1 was characterized by cytoplasmic staining of the carcinoma tissues. To quantify Gao expression, semi-quantitative scoring was performed for the evaluation of the intensity ( 0 , no staining; 1 , weak staining; 2 , strong staining) and of the fraction of positively stained cancer cells $(0$, no staining; 1 , less than half; 2 , more than half). The final score was counted for each sample by multiplying the intensity and the percentage of posi- tively stained cells $(0$, no staining; 1 , weak staining; 2 , moderate staining; 4, strong staining). Sections with a score of 0 or 1 were classified as having a low expression of Gao, whereas those with a score of 2 or 4 were classified as having a high expression or overexpression of Gao. Samples were scored by two independent researchers, neither of whom had knowledge or information pertaining to the clinical status of the patients. Immunohistochemical analysis and clinical data collection were performed independently and in a blinded manner.

Cell culture. The human GC cell lines, MGC-803 and AGS, were cultured in DMEM supplemented with $10 \%$ fetal bovine serum (FBS) and $50 \mathrm{U} / \mathrm{ml}$ penicillin/streptomycin (Sigma, St. Louis, MO, USA) and were incubated at $37^{\circ} \mathrm{C}$ in a humidified atmosphere with $5 \% \mathrm{CO}_{2}$. The medium was changed every 2-3 days.

Small interfering RNA (siRNA) and cell transfection. Two pairs of siRNA for GNAO1 (siGNAO1-1 and siGNAO1-2) were purchased from Shanghai GenePharma Co., Ltd. (Shanghai, China) and introduced into the cells using Lipofectamine 2000 (Invitrogen Life Technologies, Carlsbad, CA, USA) according to the manufacturer's instructions. In brief, the cells were seeded and incubated to reach $80 \%$ confluence prior to transfection. Four hours after transfection, the medium was replaced with full medium. After $72 \mathrm{~h}$, the cultured cells were split for protein extraction. The total protein concentration in the cell lysates was determined using the Enhanced BCA Protein Assay kit (Beyotime, Shanghai, China). The GNAO1 siRNA sequences are as follows: 5'-CCCACUUCACAUUC AAGAATT-3' and 5'-GGGCAUCGAAUAUGGUGAUTT-3'. The negative control siRNA (corresponding to non-silencing negative control siRNA) sequence was: 5'-UUCUCCGAACGU GUCACGUTT-3'.

Cell proliferation assay. Cell proliferation was measured using the Cell Counting kit (CCK)-8 and colony formation assay. CCK-8 assay was performed using the CCK-8 kit (Beyotime). According to the manufacturer's instructions, $1-5 \times 10^{3}$ cells were seeded into 96-well culture plates and allowed to attach for $24 \mathrm{~h}$. The cells were then transfected with the indicated concentrations of the negative control or GNAO1 siRNA and incubated for 24, 48, 72 or $96 \mathrm{~h}$. At the endpoint, $20 \mu \mathrm{l} \mathrm{CCK-8}$ $(5 \mathrm{~g} / \mathrm{l})$ were added for a further $4 \mathrm{~h}$. A Multiscan GO spectrophotometer (Thermo Scientific, Waltham, MA, USA) was used to measure the absorbance at $450 \mathrm{~nm}$.

According to the colony formation assay, a total of $0.5-1 \times 10^{3}$ cells was plated in 6 -well plates and transfected with siRNA. Following incubation for an additional 10-14 days, the cells were fixed with methanol and stained with $0.1 \%$ crystal violet (Sigma). Colonies of $>50$ cells were manually counted. The experiments were repeated at least 3 times.

Determination of apoptosis by flow cytometric analysis. The AGS and MGC-803 cells were transfected with siRNA targeting GNAO1 for $24 \mathrm{~h}$. The cells were harvested and washed twice with cold PBS and resuspended in binding buffer. After Annexin V-FITC labeling and incubation for $10 \mathrm{~min}$ at room temperature, the cell suspensions were immediately used in flow cytometric analysis. All experiments were performed in triplicate. 
Table I. Demographic data and survival in different stages of gastric cancer according to the AJCC classification system.

\begin{tabular}{lccccc}
\hline Variable & $\begin{array}{c}\text { Stage I } \\
\mathrm{n}=11\end{array}$ & $\begin{array}{c}\text { Stage II } \\
\mathrm{n}=38\end{array}$ & $\begin{array}{c}\text { Stage III } \\
\mathrm{n}=17\end{array}$ & $\begin{array}{c}\text { Stage IV } \\
\mathrm{n}=4\end{array}$ & $\begin{array}{c}\text { Total } \\
\mathrm{n}=70\end{array}$ \\
\hline $\begin{array}{l}\text { Gender } \\
\text { Male }\end{array}$ & 9 & & & & \\
$\quad$ Female & 2 & 26 & 14 & 1 & 50 \\
Age (years) & $68.4(13.4)$ & $67.3(13.3)$ & $78.76(12.5)$ & $59.3(11.0)$ & $69.8(13.9)$ \\
$\begin{array}{l}\text { Follow-up period } \\
\text { (months) }\end{array}$ & $55.2(18.9)$ & $38.3(22.0)$ & $33.0(22.6)$ & $30.8(22.0)$ & $38.0(22.3)$ \\
$\begin{array}{l}\text { Survival } \\
\text { Yes }\end{array}$ & 0 & 13 & 9 & 4 & 26 \\
No & 11 & 25 & 8 & 0 & 44 \\
\hline
\end{tabular}

a Data pertaining to age and follow-up period are the means \pm SD. AJCC, American Joint Committee on Cancer.

Western blot analysis. The cells were harvested and washed twice with ice-cold PBS. Cell lysates were obtained using RIPA lysis buffer supplemented with $1 \mathrm{mM}$ PMSF (both from Beyotime), and were then subjected to centrifugation at $12,000 \mathrm{x} \mathrm{g}$ for $5 \mathrm{~min}$ at $4^{\circ} \mathrm{C}$. Total protein concentrations in the supernatant were determined by the bicinchoninic acid assay (BCA; Beyotime). In the current study, experimental and control cells were used to examine the expression of GNAO1, that of cleaved apoptosis-associated PARP, as well as that of some pro-apoptotic proteins, including Bim and Puma. Total protein extracts were separated on $10-12 \%$ SDS-PAGE (20-50 mg/lane), and electrotransferred onto polyvinylidene fluoride membranes. After the transfer, the membranes were blocked for $1 \mathrm{~h}$ at room temperature with milk and then incubated overnight at $4{ }^{\circ} \mathrm{C}$ with anti-GNAO1 (1:500; Proteintech), anti-PARP (1:1,000), anti-Puma $(1: 1,000)$ (they were both purchased from Cell Signaling Technology, Danvers, MA, USA), anti-extracellular signal-regulated kinase 1 and 2 (ERK1/2; 1:1,000; Epitomics, Burlingame, CA, USA), phospho-stress-activated protein kinase (SARK)/c-Jun N-terminal kinase (JNK; 1:1,000), phosphop38 mitogen-activated protein kinase (MAPK; 1:1,000) (they were both purchased from Cell Signaling Technology) or anti- $\beta$-tubulin $(1: 2,000$; Epitomics) antibodies. The membranes were then washed 3 times with TBST (Bioscience, Shanghai, Inc., China) and incubated with anti-rabbit IgG, HRP-linked antibody (1:2,000; Cell Signaling Technology) for $2 \mathrm{~h}$ at room temperature. Washes were repeated another 3 times with TBST and immunoreactivity was determined using the Chemiluminescence Imaging LAS-3000 system (Fujifilm, Tokyo, Japan). Antibodies were diluted using the SignalBoost ${ }^{\mathrm{TM}}$ Immunoreaction Enhancer kit (Calbiochem, La Jolla, CA, USA), while $\beta$-tubulin served as an internal control.

Statistical analysis. Several clinicopathological parameters were examined: age, gender, depth of invasion, nodal status, stage, degree of differentiation, tumor size and rates of relapse. The correlation between Gao expression and each clinicopathological characteristic was examined using the $\chi^{2}$ test. The time-to-event endpoints for Gao expression were plotted by the Kaplan-Meier method, and the degree of significance was calculated by the univariate log-rank test. Parameters that emerged as significant $(\mathrm{P}<0.05)$ in univariate analysis were entered as variables in the multivariate Cox regression model, and the hazard ratio (HR) and independent prognostic impact were determined in a stepwise backward manner. The data of from the cellular experiments are expressed as the means $\pm \mathrm{SD}$. The independent samples t-test and analysis of variance (ANOVA) were used to compare data between different groups. All data were analyzed using SPSS software version 13.0. A P-value of 0.05 was considered to indicate a statistically significant difference.

\section{Results}

Clinicopathological characteristics of the recruited patients. The clinicopathological characteristics of the 70 patients with GC are presented in Table I. There were 50 males with a median age of 73 years (range, 43-102) and 20 females with a median age of 70 years (range, 45-85). The American Joint Committee on Cancer (AJCC) staging system was used to stage the primary GC samples; 11, 38, 17 and 4 patients were classified as having stage I, II, III and IV tumors, respectively. The mean duration of follow-up was 38.0 months (median, 35 months; range, 4-78 months).

The expression panel of GNAOI in GC. To characterize Gao expression in human GC, 70 archival paraffin-embedded tissue sections were used for immunohistochemistry. Representative images of GNAO1-negative (scores of 0 or 1) and -positive (scores of $\geq 2$ ) expression are presented in Fig. 1. The cytoplasmic expression of $\mathrm{G} \alpha$ o (scores of $\geq 1$ ) was clearly observed in all GC tissues and in parts of the corresponding adjacent tissues (7/26, 26.9\%). Goo was overexpressed (scores of 2 or 4 ) in the cancer tissues from 44 of 70 patients (62.9\%) and in none of the corresponding adjacent tissues. It was further revealed that the expression of Gao correlated with tumor size $(\mathrm{P}=0.003)$, the degree of histological differentiation $(\mathrm{P}=0.025)$ and TNM stage $(\mathrm{P}=0.004)$. Of note, the Gao staining index did not present any statistically significant difference between 

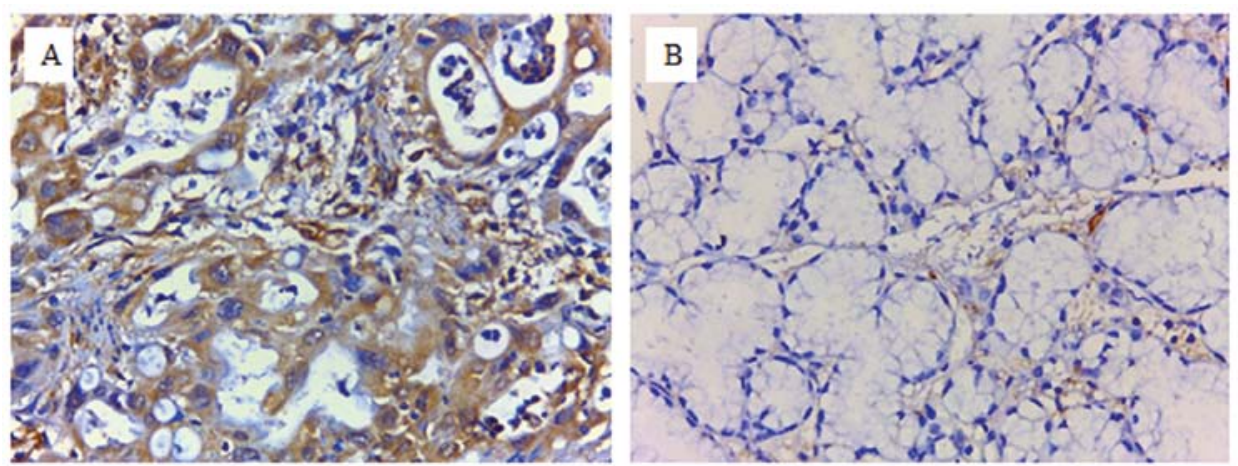

Figure 1. (A) The expression of guanine nucleotide-binding protein $\mathrm{G}(\mathrm{O})$ subunit alpha (GNAO1) in gastric cancer tissue and (B) the adjacent non-cancerous tissue, from the same subject detected by immunohistochemistry. Original magnification, x200.

Table II. GNAO1 expression in gastric cancer and its correlation with clinicopathological parameters.

\begin{tabular}{|c|c|c|c|c|}
\hline \multirow[b]{2}{*}{ Variable } & \multirow[b]{2}{*}{$\mathrm{n}$} & \multicolumn{2}{|c|}{ GNAO1 expression score } & \multirow[b]{2}{*}{ P-value } \\
\hline & & $\begin{array}{l}0 \text { or } 1 \\
n=26\end{array}$ & $\begin{array}{l}2 \text { or } 4 \\
n=44\end{array}$ & \\
\hline \multicolumn{5}{|l|}{ Age (years) } \\
\hline$\geq 66$ & 21 & 10 & 11 & 0.359 \\
\hline$<66$ & 49 & 16 & 33 & \\
\hline \multicolumn{5}{|l|}{ Gender } \\
\hline Male & 50 & 18 & 32 & 0.969 \\
\hline Female & 20 & 8 & 12 & \\
\hline \multirow{2}{*}{\multicolumn{5}{|c|}{$\begin{array}{l}\text { Tumor histology } \\
\text { Degree of } \\
\text { differentiation }\end{array}$}} \\
\hline & & & & \\
\hline Poor & 33 & 11 & 23 & 0.025 \\
\hline Moderate & 6 & 0 & 6 & \\
\hline Well & 0 & 0 & 0 & \\
\hline Unknown & 31 & 15 & 16 & \\
\hline \multicolumn{5}{|c|}{$\begin{array}{l}\text { Lymph node } \\
\text { involvement }\end{array}$} \\
\hline Absent & 21 & 6 & 15 & 0.483 \\
\hline Present & 49 & 20 & 29 & \\
\hline \multicolumn{5}{|l|}{ Stage } \\
\hline I & 11 & 4 & 7 & 0.004 \\
\hline II & 38 & 15 & 23 & \\
\hline III & 17 & 5 & 12 & \\
\hline IV & 4 & 2 & 2 & \\
\hline \multicolumn{5}{|l|}{ Tumor size } \\
\hline$\geq 3 \mathrm{~cm}$ & 44 & 17 & 27 & 0.006 \\
\hline$<3 \mathrm{~cm}$ & 26 & 9 & 17 & \\
\hline
\end{tabular}

${ }^{a}$ All statistical tests were two-tailed and a value of $\mathrm{P}<0.05$ was considered to indicate a statistically significant difference. GNAO1, Guanine nucleotide-binding protein $\mathrm{G}(\mathrm{O})$ subunit alpha.

tumors obtained from patients with different age, gender, family history or lymph node involvement (Table II).



Figure 2. Survival analysis for 70 patients, stratified by guanine nucleotidebinding protein $\mathrm{G}(\mathrm{O})$ subunit alpha (GNAO1) immunoreactivity [GNAO1(-): score, 0 or 1; GNAO1(+): score, 2 or 4]. All statistical tests were two-tailed and the significance level was set at 0.05 .

Prognostic significance of GNAO1 expression in GC. To evaluate whether GNAO1 expression can be used as a prognostic factor, we performed survival analysis using the Kaplan-Meier method. The overall survival curves in relation to GNAO1 expression were statistically significant $(\mathrm{P}=0.033$; Fig. 2$)$. The mean survival was 55.0 months (median, 61 months) in the GNAO1-negative group and 27.7 months (median, 28 months) in the GNAO1-positive group. The prognostic significance of GNAO1 expression in GC was further confirmed by univariate survival analysis $(\mathrm{P}<0.05)$. After the adjustment of other prognostic indicators by multivariate analysis, GNAO1 remained an independent prognostic factor for patients with $\mathrm{GC}(\mathrm{P}=0.046)$ (Table III).

Roles of GNAOI in GC cells. To examine the possible roles of GNAO1 in GC cells, GNAO1 expression was knocked down by siRNA. The efficacy of GNAO1 siRNA was evaluated using a FAM fluorescent signal (data not shown) and western blot analysis (Fig. 3). It was found that the expression of GNAO1 was almost completely suppressed at the protein level when the AGS and MGC-803 cells were transfected with GNAO1 siRNA. CCK-8 and colony formation assays were conducted following 
Table III. Univariate and multivariate analysis of prognostic factors for survival in patients with gastric cancer.

\begin{tabular}{|c|c|c|c|}
\hline \multirow[b]{2}{*}{ Variable } & \multirow{2}{*}{$\frac{\text { Univariate analysis }}{\text { P-value }}$} & \multicolumn{2}{|c|}{ Multivariate analysis } \\
\hline & & P-value & RR $(95 \%$ CI $)$ \\
\hline \multicolumn{4}{|l|}{ Relapse } \\
\hline Yes (vs. no) & 0.039 & 0.139 & $0.535(0.234-1.224)$ \\
\hline \multicolumn{4}{|l|}{ TNM stage } \\
\hline Stage IV (vs. III + II + I) & 0.003 & 0.003 & $1.971(1.255-3.096)$ \\
\hline \multicolumn{4}{|l|}{ Tumor size } \\
\hline$\geq 3 \mathrm{~cm}($ vs. $<3 \mathrm{~cm})$ & 0.038 & 0.186 & $0.471(0.154-1.439)$ \\
\hline \multicolumn{4}{|l|}{ GNAO1 expression } \\
\hline Positive (vs. negative) & 0.045 & 0.046 & $2.766(1.018-7.518)$ \\
\hline
\end{tabular}

$\mathrm{CI}$, confidence interval; RR, relative risk; TNM, tumor-node metastasis staging system; GNAO1, Guanine nucleotide-binding protein $\mathrm{G}(\mathrm{O})$ subunit alpha.
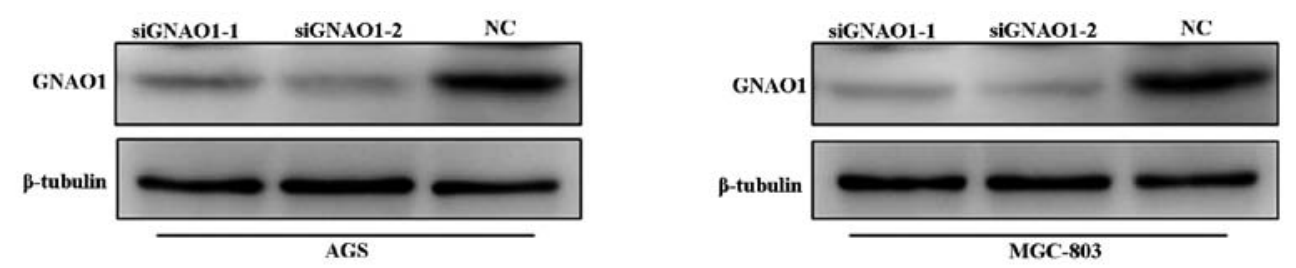

Figure 3. The efficacy of guanine nucleotide-binding protein $\mathrm{G}(\mathrm{O})$ subunit alpha (GNAO1) siRNA was detected by western blot analysis. When the AGS and MGC-803 cells were transfected with GNAO1 siRNA, the expression of GNAO1 was almost completely suppressed at the protein level.
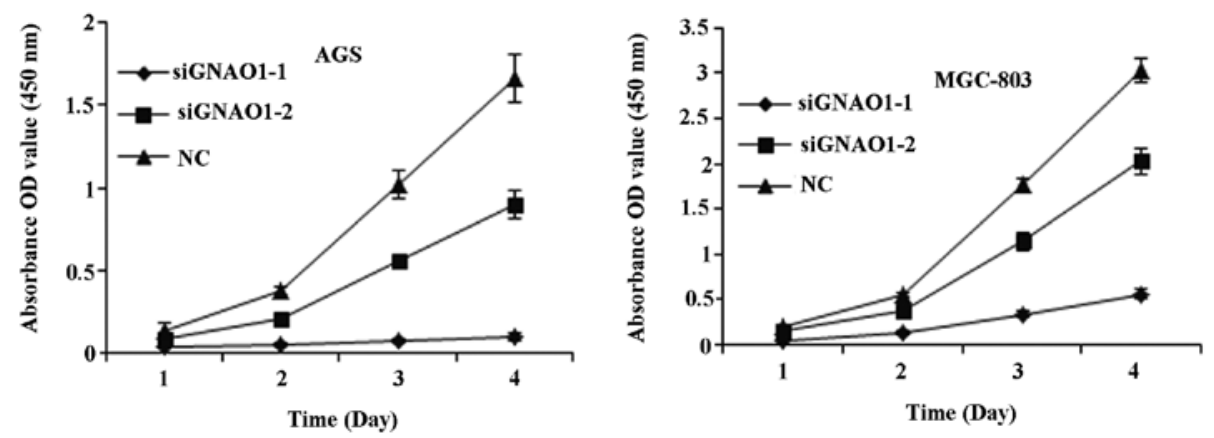

Figure 4. The proliferation of gastric cancer cells was determined by CCK-8 assay 1-4 days after transfection with small interfering RNA (siRNA) and was significantly reduced $(\mathrm{P}<0.05)$ following transfection with siRNA. Statistical analysis was performed with data from 5 individuals. NC, negative control; GNAO1, guanine nucleotide-binding protein $\mathrm{G}(\mathrm{O})$ subunit alpha.

transfection with siRNA and revealed that GNAO1 siRNA significantly inhibited cell proliferation $(\mathrm{P}<0.01)($ Fig. 4$)$ and the colony formation ability of the cells $(\mathrm{P}<0.05)$ (Fig. 5).

We also examined the effects of GNAO1 on the apoptosis of GC cells by flow cytometry. Flow cytometric analysis using Muse Annexin V and Dead Cell kit (Millipore) revealed that the percentage of early, late and total apoptotic, as well as dead cells markedly increased $48 \mathrm{~h}$ after transfection with GNAO1 siRNA (Fig. 6).

Induction of apoptosis-associated mitochondrial events in GC cells by GNAO1 knockdown and the involvement of the ERK signaling pathway. To confirm that GNAO1 participates in the regulation of the proliferation and apoptosis of GC cells and to determine the possible signaling pathways involved, we examined the expression of the apoptosis-related protein, PARP, and that of the pro-apoptotic proteins, Bim and Puma, as well as the activation of ERK1/2 and phospho-SARK/JNK following transfection with siRNA. It was found that the abundance of PARP, Puma and Bim was increased after GNAO1 knockdown (Fig. 7). In addition, the phosphorylation levels of ERK were markedly decreased in response to treatment, while no significant changes were observed in the phosphorylation levels of SARK/JNK and p38 (Fig. 8).

\section{Discussion}

GC remains a major public health issue worldwide (16). Although it is curable if detected early, the majority of patients are diagnosed at an advanced stage and have a poor prog- 



siGNAO1-1
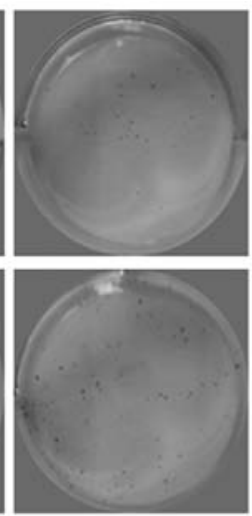

siGNAO1-2
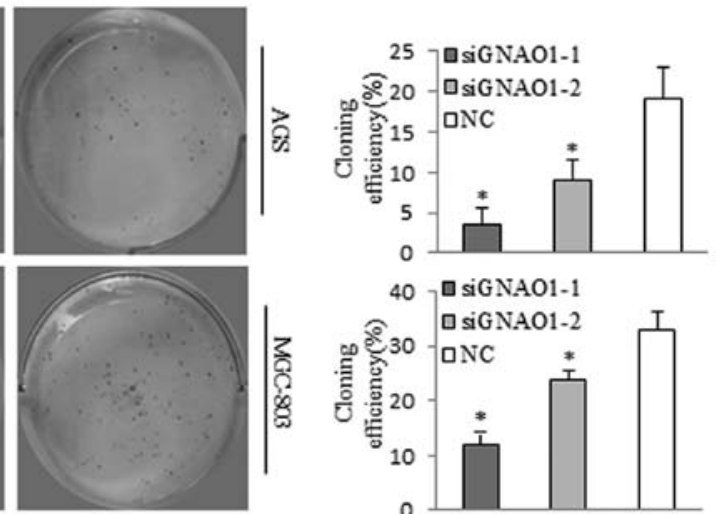

$\mathrm{NC}$

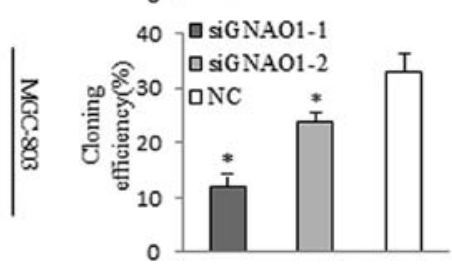

Figure 5. The tumorigenicity of gastric cancer (GC) cells was semi-quantified by colony formation assay. The tumorigenicity of GC cells was significantly decreased ("P<0.01) following transfection with siRNA. Statistical analysis was performed with data from three individuals. NC, negative control; GNAO1, guanine nucleotide-binding protein $\mathrm{G}(\mathrm{O})$ subunit alpha.

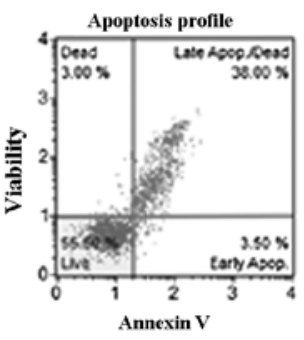

siGNA01-1

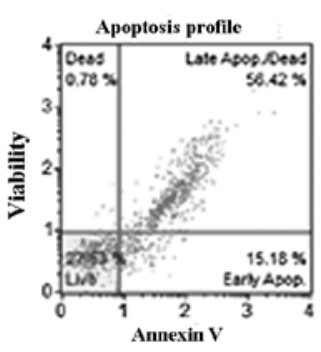

siGNAO1-1

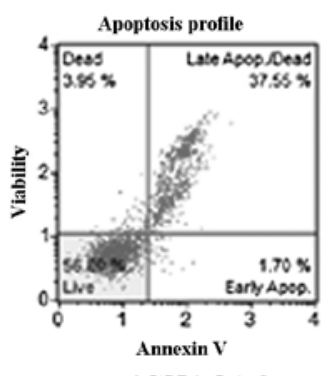

siGNAO1-2

AGS

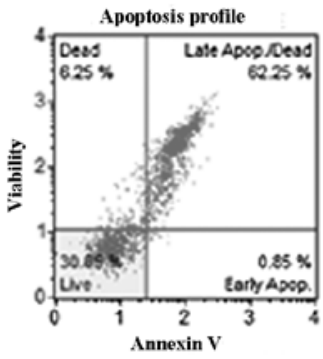

siGNAO1-2

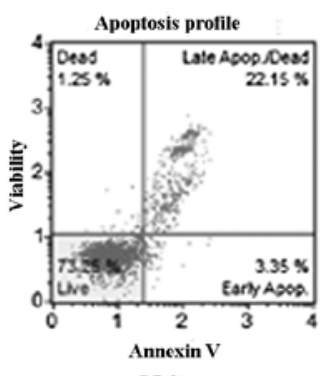

NC
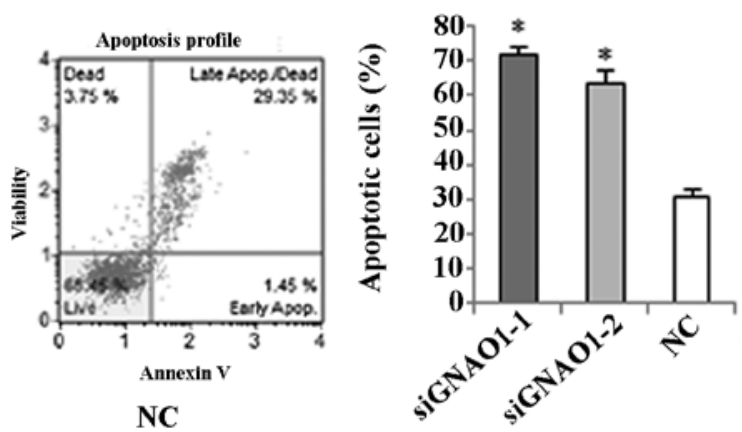

Figure 6. The anti-apoptotic effect of guanine nucleotide-binding protein $\mathrm{G}(\mathrm{O})$ subunit alpha (GNAO1) was detected by flow cytometric analysis with Muse Annexin V and the Dead Cell kit. The percentage of apoptotic cancer cells was significantly increased (" $\mathrm{P}<0.05)$ in the siRNA-treated groups.

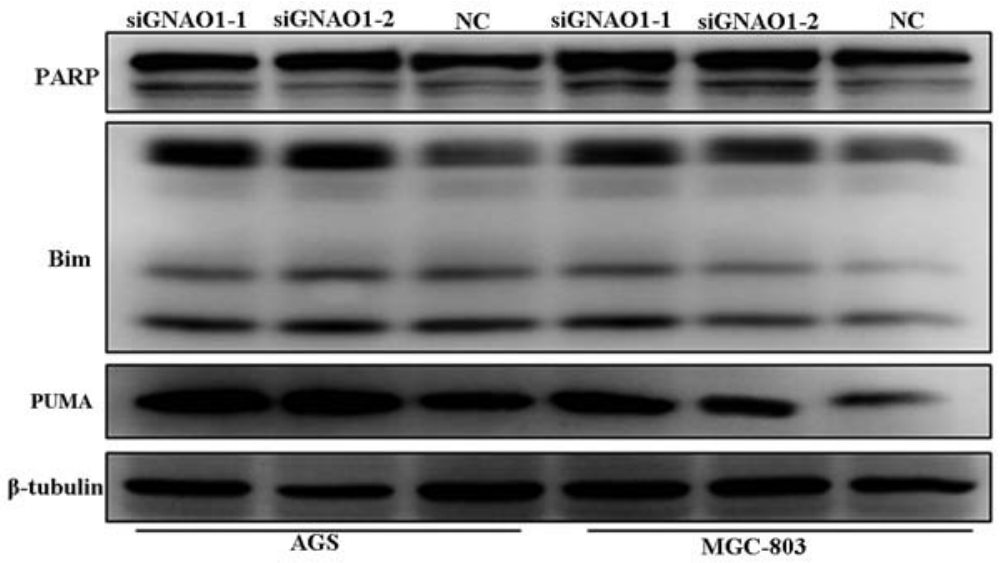

Figure 7. Western blot analysis of apoptosis-associated proteins. The expression levels of PARP, Puma and Bim were increased after GNAO1 knockdown. GNAO1, guanine nucleotide-binding protein $\mathrm{G}(\mathrm{O})$ subunit alpha. 

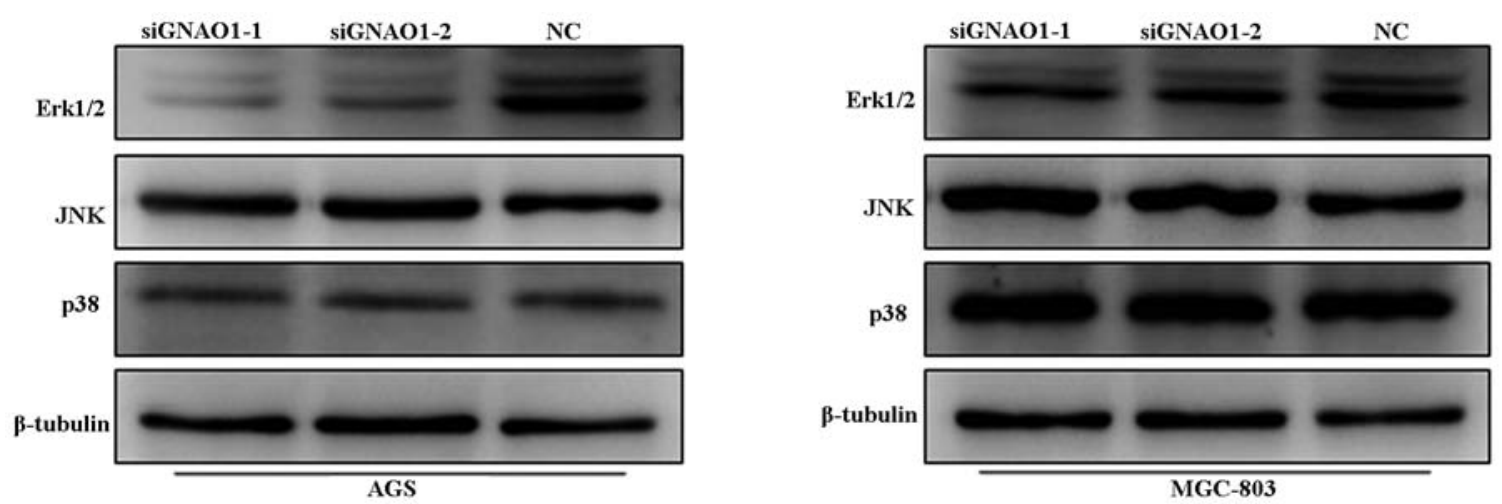

Figure 8. Western blot analysis of the phosphorylation level of ERK, SARK/JNK and p38 MAPK. The phosphorylation levels of ERK were markedly decreased in response to transfection with GNAO1 siRNA. No significant changes in the phosphorylation levels of SARK/JNK and p38MAPK were observed following transfection with siRNA. GNAO1, guanine nucleotide-binding protein $\mathrm{G}(\mathrm{O})$ subunit alpha.

nosis (17). The precise prediction of the risk of recurrence would assist in minimizing the adverse effects of GC and maximizing the therapeutic effects of treatment. Therefore, greater knowledge of the molecular mechanisms underlying the development of this deadly neoplasm is required if novel valuable predictors of prognosis for patients with GC are to be developed. It is well known that tumors are a result of uncontrolled cell proliferation in different organs. The disruption of the balance between cell proliferation and apoptosis is an important driving force in the development of GC (18). Proliferation markers have been widely used in order to diagnose and determine the behavior and prognosis of benign and malignant tumors (19).

Based on the present study, we hypothesized that GNAO1 may be a novel marker of proliferation for GC. Studies have demonstrated that the GNAO1 gene is frequently mutated or absent in many types of human primary carcinomas. However, few studies have investigated the expression and biological function of the GNAO1 gene. To the best of our knowledge, there are no published reports to date that discuss the prognostic significance of GNAO1 in human cancer, including GC. In the current study, using samples from 70 patients with GC, we demonstrate that GNAO1 is overexpressed in GC tissues and that its upregulation is inversely correlated with patient survival. GNAO1 can also be used to predict poorer outcomes for patients with GC. GNAO1 overexpression appears to be a useful marker for predicting the outcome in patients with GC who have undergone surgery to remove the tumor. Thus, patients with GC who present with an overexpression of GNAO1 should perhaps be followed-up more closely. Future studies with larger GC patient groups are recommended to confirm the prognostic significance of GNAO1 in this disease.

To further explain these observations, we also examined the role of GNAO1 in vitro and concluded that GNAO1 may participate in the regulation of GC cell proliferation by affecting cell apoptosis through the accumulation of apoptotic proteins, including PARP, Puma and Bim, which may be partially regulated by ERK inactivation.

It is well known that the cleavage of PARP is a characteristic of apoptosis and that the $\mathrm{BH} 3$ domain-containing proteins, Bim and Puma, can lead to apoptosis (20). The results of the present study demonstrated the accumulation of Puma, Bim and PARP after the knockdown of the GNAO1 gene
(Fig.7). In conjunction with the apoptotic effect of GNAO1 siRNA demonstrated by flow cytometry, these findings indicate that GNAO1 expression inhibits the apoptosis of GC cells. It has recently been shown that $\mathrm{G} \alpha$ o overexpression results in the increased phosphorylation and hence in the activation of ERK1/2 but not in that of p38 or JNK (21). MAPKs, which consist of these 3 protein kinases $(22,23)$, have been reported to regulate diverse cellular functions, including embryogenesis, proliferation, differentiation and apoptosis (24). In addition, previous studies have indicated that the inhibition of the ERK pathway induces an increase in Bim expression levels and activates the Puma promoter. Consequently, it can be identified as the tunnel that interconnects kinase signaling networks and the mitochondrion-dependent apoptotic program (25-27). Thus, in this study, we investigated the activity of ERK1/2, SARK/JNK and p38 MAPK after GNAO1 silencing. Confirming our initial hypothesis, we found that ERK activation may be involved in the downregulation of Bim and Puma, as well as in the inhibition of the apoptosis of GC cells by GNAO1.

To the best of our knowledge, in the current study we demonstrate for the first time that GNAO1 overexpression in GC is associated with tumor size, tumor differentiation, TNM stage and poor prognosis. The present findings also demonstrate that the knockdown of GNAO1 leads to reduced proliferation and promotes the apoptosis of GC cells (MGC-803 and AGS). Thus, GNAO1 may serve as a novel diagnostic and prognostic biomarker, as well as a potential therapeutic target in GC.

\section{Acknowledgements}

This study was supported by grants from the National Natural Science Foundation of China (nos. 81125001 and 91129702), the Ministry of Science and Technology of China (no. 2010CB732405) and the Science and Technology Commission of Shanghai Municipality (nos. 12JC1402000 and 12410705300).

\section{References}

1. Nakamura T, Nakatsu N, Yoshida Y, et al: Identification of candidate genes determining chemosensitivity to anti-cancer drugs of gastric cancer cell lines. Biol Pharm Bull 32: 1936-1939, 2009. 
2. Ji YB, Qu ZY and Zou X: Juglone-induced apoptosis in human gastric cancer SGC-7901 cells via the mitochondrial pathway. Exp Toxicol Pathol 63: 69-78, 2011.

3. Jackson C, Cunningham D and Oliveira J: Gastric cancer: ESMO clinical recommendations for diagnosis, treatment and follow-up. Ann Oncol 20 Suppl 4: 34-36, 2009.

4. Wang WF, Li J, Du LT, et al: Krüppel-like factor 8 overexpression is correlated with angiogenesis and poor prognosis in gastric cancer. World J Gastroenterol 19: 4309-4315, 2013.

5. Yamada Y, Boku N, Nishina T, et al: Impact of excision repair cross-complementing gene 1 (ERCC1) on the outcomes of patients with advanced gastric cancer: correlative study in Japan Clinical Oncology Group Trial JCOG9912. Ann Oncol 24 2560-2565, 2013.

6. Li X, Qi W, Yao R, Tang D and Liang J: Overexpressed transcription factor FOXM1 is a potential diagnostic and adverse prognostic factor in postoperational gastric cancer patients. Clin Transl Oncol: Jul 20, 2013 (Epub ahead of print)

7. Kamangar F, Dores GM and Anderson WF: Patterns of cancer incidence, mortality, and prevalence across five continents: defining priorities to reduce cancer disparities in different geographic regions of the world. J Clin Oncol 24: 2137-2150, 2006

8. Neer EJ, Lok JM and Wolf LG: Purification and properties of the inhibitory guanine nucleotide regulatory unit of brain adenylate cyclase. J Biol Chem 259: 14222-14229, 1984.

9. Gierschik P, Milligan G, Pines M, et al: Use of specific antibodies to quantitate the guanine nucleotide-binding protein Go in brain Proc Natl Acad Sci U S A 83: 2258-2262, 1986.

10. Corre I, Baumann H and Hermouet S: Regulation by Gi2 proteins of v-fms-induced proliferation and transformation via Src-kinase and STAT3. Oncogene 18: 6335-6342, 1999.

11. Fan X, Brass LF, Poncz M, Spitz F, Maire P and Manning DR: The alpha subunits of $\mathrm{Gz}$ and $\mathrm{Gi}$ interact with the eyes absent transcription cofactor Eya2, preventing its interaction with the six class of homeodomain-containing proteins. J Biol Chem 275 32129-32134, 2000.

12. Montminy M: Transcriptional regulation by cyclic AMP. Annu Rev Biochem 66: 807-822, 1997.

13. Kan Z, Jaiswal BS, Stinson J, et al: Diverse somatic mutation patterns and pathway alterations in human cancers. Nature 466 869-873, 2010.
14. Jia D, Wei L, Guo W, et al: Genome-wide copy number analyses identified novel cancer genes in hepatocellular carcinoma. Hepatology 54: 1227-1236, 2011.

15. Garcia-Marcos M, Ghosh P and Farquhar MG: Molecular basis of a novel oncogenic mutation in GNAO1. Oncogene 30 2691-2696, 2011.

16. Jemal A, Bray F, Center MM, Ferlay J, Ward E and Forman D: Global cancer statistics. CA Cancer J Clin 61: 69-90, 2011.

17. Wang $\mathrm{XN}$ and Liang $\mathrm{H}$ : Some problems in the surgical treatment of gastric cancer. Chin J Cancer 29: 369-373, 2010.

18. Nguyen DX, Bos PD and Massagué J: Metastasis: from dissemination to organ-specific colonization. Nat Rev Cancer 9: 274-284, 2009.

19. Freeman A, Morris LS, Mills AD, et al: Minichromosome maintenance proteins as biological markers of dysplasia and malignancy. Clin Cancer Res 5: 2121-2132, 1999.

20. Tait SW and Green DR: Mitochondria and cell death: outer membrane permeabilization and beyond. Nat Rev Mol Cell Biol 11: 621-632, 2010.

21. Bratton MR, Antoon JW, Duong BN, et al: Goo potentiates estrogen receptor $\alpha$ activity via the ERK signaling pathway. J Endocrinol 214: 45-54, 2012.

22. Baines CP and Molkentin JD: STRESS signaling pathways that modulate cardiac myocyte apoptosis. J Mol Cell Cardiol 38: 47-62, 2005.

23. Fan Y, Chen H, Qiao B, et al: Opposing effects of ERK and p38 MAP kinases on HeLa cell apoptosis induced by dipyrithione. Mol Cells 23: 30-38, 2007.

24. Raman M, Chen W and Cobb MH: Differential regulation and properties of MAPKs. Oncogene 26: 3100-3112, 2007.

25 . Youle RJ and Strasser A: The BCL-2 protein family: opposing activities that mediate cell death. Nat Rev Mol Cell Biol 9: 47-59, 2008.

26. Balmanno K and Cook SJ: Tumour cell survival signalling by the ERK1/2 pathway. Cell Death Differ 16: 368-377, 2009.

27. Bean GR, Ganesan YT, Dong Y, et al: PUMA and BIM are required for oncogene inactivation-induced apoptosis. Sci Signal 6: ra20, 2013. 